and nurses, long contract staff, male aged below 30. Persons who were apt to commit workplace violence were relatives of patients, aged below 40, especially male aged 31-40. Wards and day shift were the most likely location and time slot for violence.

Conclusion Prevalence of workplace violence in hospitals in South China was quite high. Staff in Emergency Departments, psychiatrists, doctors and nurses, long contract staff and male aged below 30 should be put more emphasis on from workplace violence. Prevention and control of violence was a complex and systematic project, calling for collaboration from various sectors such as law enforcement, management, engineering, education and so forth.

\section{PREVALENCE OF AND RISK FACTORS ASSOCIATED WITH WORKPLACE VIOLENCE: A CROSS-SECTIONAL STUDY IN 7026 HEALTH STAFF IN SOUTH CHINA}

Z Chen*, C Peek-Asa, G Yang Correspondence: The First Affiliated Hospital of Jinan University, West No 613 Huangpu Road Tianhe District, Jinan University Guangzhou, Guangdong 510630, China

10.1136/ip.2010.029215.13

Objective To probe prevalence of and risk factors associated with workplace violence in hospitals so as to establish a theory basis for maintaining regular working order in hospitals and lawmaking against workplace violence.

Method A retrospective study about workplace violence in health staff from four different cities of South China was carried out, utilizing SPSS 17.0 for regressive and GEE analysis.

Results Among 7026 hospital staff investigated, 4075 have been subjected to workplace violence in the last 12 months preceding the survey, with a rate of $57.93 \%$, accounting for $56.76 \%$ of psychological violence, $15.06 \%$ of physical violence and $6.67 \%$ of sexual violence, respectively. Victims differed in four cities, with rates varying from $28.68 \%$ to $66.20 \%$. Staff who were most prone to violence in hospitals were those working at Emergency Departments, psychiatrists, doctors 